\title{
AGAPE ALS GRUNDBEGRIFF DES CHRISTLICHEN ETHOS Biblisch-strukturelle Analysen
}

Es gibt Versuche, das christliche Ethos an die Idee der Berufung oder der Nachahmung von Christi (F. Tillmann) oder Gottesbilds anzulehnen ${ }^{1}$. Dagegen wird das Gebot der Liebe als Ausgangspunkt der Ethik des Neuen Testaments verhältnismässig selten unterstrichen, obwohl es schon ziemlich viel zum Begriff der Agape geschrieben worden ist ${ }^{2}$. Die Ursache dessen ist in der noch nicht ausreichenden Bearbeitung des Begriffs der Agape selbst zu sehen. Schon A. Nygren macht in seinem bekannten Werk Eros und Agape ${ }^{3}$ der katholischen Auffassung der Caritas den Vorwurf, dass sie mit dem biblischen Begriff der Agape zu wenig und mit dem Eros-Motiv dafür zu sehr verbunden ist. Seinen Standpunkt, den er in den Dreissiger Jahren unseres Jahrhunderts vertrat, hatte Nygren in der neuen Ausgabe seines Werkes (1955) nicht geändert, obwohl ernsthafte Arbeiten zum Thema Agape inzwischen erschienen sind.

Es ist möglich, dass die bisherigen Forscher des Begriffs der Agape zu viel Wert auf die vergleichenden Analysen der ausserbiblischen Begriffe und Motiven legten, statt sich an der eingehenden Analyse der biblischen Begriffe selbst zu konzentrieren. Diesbezüglich eine interessante Bemerkung von einem der Kenner dieses Problems, V. Warnach ${ }^{4}$ :

${ }^{1} \mathrm{Vgl}$. Schnackenburg, La théologie du Nouveau Testament. État de la question (Studia Neotestamentica, Subsidia I), 1961, 109. Dazu: K. O k a y a ma, Zur Grundlegung christlicher Ethik. Theologische Konzeptionen der Gegenwart im Lichte des Analogie-Problems, Berlin-New York-1977.

$2 \mathrm{Vgl}$. R. Schnackenburg, Die sittliche Botschaft des Neuen Testaments, München 21962, 65-81, 172-178. Ferner: C. S p i c q, Agapè dans le Nouveau Testament. Analyse des textes, 3 Bde, Paris 1958-1959; G. O u tka, Agape, An Ethical Analysis, New Haven-London 1972; B. W elte, Dialektik der Liebe. Gedanken zur Phänomenologie der Liebe und zur christlichen Nächstenliebe im technologischen Zeitaler, Frankfurt 1975; S. L éga s s e, L'étendue de l'amour interhumain d'après le Nouveau Testament: limites et promesses, in: „Revue Théologique de Louvain" 8 (1977) 137-159; H. T i m m, Geist der Liebe: die Ursprungsgeschichte der religiösen Anthropotheologie, Gütersloh 1978; G. G hit ti, L'amore agape come espressione della maturita personale del cristiano. Una interpretazione esistenziale dell'amore del prossimo, Brixiae 1978.

3 Berlin 1955. (1. schwedische Auflage: Stockkholm I 1930, II 1936).

4 Agape. Die Liebe als Grundmotiv der neutestamentlichen Theologie, Düsseldorf (1951), 265. 
„Die oft missliche Lage der Theologie und zumal der Exegese dürfte wohl darin ihren Grund haben, dass sie sich ihre Begriffe meist aus fremdem, eben profanen Denksystemen geben liesst, anstatt sie aus den ihr allein zu Gebote stehenden untrüglichen Quellen der Offenbarung zu gewinnen. Dass dabei unsere rationalen Denkformen - wiewohl sie im philosophischen Bereich ihre relative Geltung bewahren -- gesprengt oder vielmehr positiv »aufgehoben «, eben »erfüllt « werden, kann keinen Einwand gegen ein solches Bemühen derstellen. Echt christliches Denken muss in jeder Hinsicht, auch in bezug auf die Erkenntnismittel theozentrisch sein; es ist zuerst immer katabatisch eingestellt, weil es von Gott her kommt, von Gott aus die Dinge und Menschen sieht, und dann erst versucht es die Anabasis, indem es alles wieder auf Gott zurückbezieht. Jedenfalls darf man es nicht rein anabatisch auffassen, wie es das hellenisch-abendländische Denken im allgemeinen tut. Daher ist es eine der vordringlichsten Aufgaben der Gegenwart, das theologische Denken aus dieser »babylonischen" Gefangenschaft und Sprachverwirrung zu befreien".

Daher scheint es bei der Betrachtung des Themas der Agape erstrebenswert, einigen ethischen Modellen, die sich im Neuen Testament an das Agape-Prinzip anlehnen, unsere Aufmerksamkeit zu schenken. Das Ziel der vorliegenden Abhandlung wird die Darstellung des ethischen Modells der Agape bei Johannes. Wir wollen es zuerst anhand der Darstellung der semantischen Entwicklung des Begriffs der Agape schildern.

\section{DIE SEMANTISCHE ENTWICKLUNG DER AGAPE}

Die systematischen lexikograpischen Untersuchungen an dem Begriff der Agape wurden von C. Spicq gründlich durchgeführt ${ }^{5}$. Indem sich Spicq auf den fragmentarischen Forschungen seiner Vorgänger $(\mathrm{H}$. Riesenfeld, A. Ceresa-Gastaldo) stützte, behauptete er, dass das Verbum agapan, das in der klassischen griechischen Sprache eine eher zweittrangige Rolle spiellte, in dem Wortschatz der Septuaginta und bei den antiken Autoren eine führende Stellung einnahm und die anderen synonimischen Verben wie: stergein, eran oder gar philein verdrängte. Spicq machte auf die semantische Entwicklung des Termins agapan aufmerksam: während es bei Homer die Zärtlichkeit, Zartheit bezeichnete, sind bei den attischen Rednern Neubedeutungen bewertenden Charakters bemerkbar, „Der Typ agapan - schrieb Spicq - ist die Mutterliebe,

5 Agapè. Prolégomènes a une étude de théologie néo-testamentaire (Studia hellenistica 10), Louvain 1955. 
die eine reine Gabe ist, es ist auch die Fähigkeit der Anpassung, die Zärtlichkeit und Empfindlichkeit des Verstehens und dadurch jegliche Einigkeit und Verschmelzung mit dem Objet der Liebe" .

Das Substantiv agapē betreffend, sind die Forscher zum Problem seiner Genese nicht einig. Es ist die Meinung vorherrschend, dass agape im Unterschied zum agapēsis - von der Septuaginta gewählt wurde, weil es noch nich all die Konotationen besass, die schon in der heidnischen Literatur zur Bezeichnung der Liebe synonimisch funktionierten. Jedenfalls vertritt man die Meinung, dass das Substantiv agape in den jüdisch-hellenistischen Kreisen noch vor der christlichen Ära aufgetaucht is und von der Septuaginta und den christlichen Autoren zuerst in Ägypten aufgenommen wurde. Die Krönung der Entwicklung des semantischen Prozesses der Gruppe agapan - agapē war also der christliche Usus, vor allem im Neuen Testament.

Die reueren Forschungen vom R. Jolly ${ }^{7}$ ändern gewissermassen die bisherigen Auffassungen. Im Lichte dieses Forschungen erweist sich nämlich, dass die Entwicklung des Wortes agapē noch vor der christlichen Ära anzusetzen ist, und zwar begann das Wort philein schon seit dem 4. Jh. von Chr. die Bedeutung ,ich liebe" zu verlieren, um sich auf die Bedeutungen ,ich umarme”, ,ich drücke” zu beschränken. Daher erschien immer häufiger das Verb agapan und verdrängte die übrigen Synonyme. Dieser Vorgang hat viel früher als man bisher dachte eingesetzt und dies im Bereich der heidnischen Literatur, was sich am Beispiel von Polibius beobachten lässt.

Ist also die lexikalische Gruppe agapan - agapē in der christlichen Literatur gar nicht originell?

Dieses Problem muss unter einem anderen Gesichtspunkt betrachtet werden, und zwar nicht nur von dem lexikographischen her - wie es bei Spicq der Fall ist - sondern anhand der strukturellen Analyse. Das zu untersuchende Wort, bzw. der Begriff darf nich vom seinem Kontext isoliert werden. In solchem Falle dürften wir natürlich nicht von der christlichen Eigentümlichkeit der Lexik agapan - agapē sprechen. Das zu untersuchende Wort soll in dem breiten Kontext seiner Distribution betrachtet werden. Die Originalität der Lexik wurzelt (nach J. Barr ${ }^{8}$ ) eher in der neuen Kombination der Wörter, in der das gegebene Wort entweder seinem ursprünglichen Sinn bewahrt, oder einen neuen

6 Ebenda 58.

7 Le vocabulaire chrétien de l'amour est-il original?, Bruxelles 1968. Vgl. hierzu: O. W is ch me ye r, Agape in der ausserchristlichen Antike, in: „Zeitschrift für die Neutestamentliche Wissenschaft und die Kunde der älteren Kirche" 69 (1978) 212-238.

8 The Semantics of the Biblical Language, Oxford 1961, 263. Vgl. auch D. Hill, Greek Words and Hebrew Meanings, Cambridge 1967, 1-22. 
bekommt. Die zeitgenössische strukturelle Semantik (E. Benveniste, A. J. Greimas ${ }^{9}$ ) unterstreicht, dass ein Wort im Text (discours) in dem es gebraucht wird den Sinn bekommt, oder, wenn wir die Grenze der Interpretation verschieben - in dem kulturellen Kontext, in dem es auftaucht.

Es unterliegt keinem Zweifel, dass sowohl der hellenistische Judaismus als auch das Urchristentum diesen neuen kulturellen Kontext bildeten, der die Lexik agapan - agapē bereicherte und entwickelte. Daher sollen die Forschungen an dem semantischen Wert der Agape im Christentum nicht unter dem Gesichtspunkt der Bestimmung des breiten oder engen Wortsinns, sondern im Rahmen der Sprache, die sich des Begriffs bediente, vorgenommen werden.

Wollen wir also dem strukturellen Modell der Agape, das uns im Ersten Johannesbrief vorliegt, unsere strenge Aufmerksamkeit im Kontext der Agape-Doktrin des Neuen Testaments widmen ${ }^{10}$.

\section{AGAPE ALS GEBOT UND KRITERIUM DER EINIGKEIT MIT GOTT}

Nach dem 1 Joh ist die absolute Treue den Geboten und vor allem dem Gebot der brüderlichen Liebe die Bedingung für ein wahres Christentum (N. Lazure ${ }^{11}$ ). Der hl. Johannes unterstreicht den göttlichen Ursprung des Liebegebotes; wollen wir nur zwei Texte anführen:

1 Joh 4, 21: "dieses Gebot haben wir v o n i h m"

5, 2: "wenn wir Gott lieben und s e i n e G e b o t e erfüllen"

Das Wort "Gebot” (entolē) tritt im J Joh 14 Mal auf, sowohl im Singular als auch im Plural. Diese Alternation der Numeri macht uns aufmerksam. Das Gebot im Sg. drückt das Wesen aller Gebote aus. Agape ist das Zentrum und zugleich die Grundlage aller Gottesgebote. Im 1 Joh 3, 23 lesen wir: „Und das ist sein G e b ot: Wir sollen an den Namen seines Sohnes Jesus Christus glauben und einander lieben, wie es seinem Gebot entspricht". So umfasst d a s Gebot in Johannes Gedanken zwei Elemente: den Glauben an Jesus Christus und die Liebe Agape. Johannes Idee ist klar: wer di e Gebote folgen will, soll vor allem di e s e s Gebot ausführen, dass den Glauben und die Nächstenliebe regiert.

9 E. B envenis t e, Problèmes de linguistique générale, Paris 1966; A.-J. G r ei mas, Sémantique structurale. Recherche de méthode, Paris 1966.

$10 \mathrm{Vgl}$. A. Feuillet, Le mystère de l'amour divin dans la théologie johannique, Paris 1972; M. Lattke, Einheit im Wort. Die spezifische Bedeutung von „Agape”, „Agapan” und „Filein” im Johannes-Evangelium, München 1975; D. D ide berg, Saint Augustin et la première épître de Saint Jean. Une théologie de l'agapè, Paris 1975; C. S p i c q, L’amour de Dieu révélé aux hommes dans les écrits de saint Jean, Paris 1978.

11 Les valeurs morales de la théologie johannique, Paris 1965, $131 \mathrm{f}$. 
In dieser Perspektive kommt die Relation zwischen dem Glauben und der Liebe klar zutage. Das Gebot des Glaubens im Namen Jesus Christi bringt die Pflicht mit sich, an die Offenbarung Gottes und Christus Worte $\mathrm{zu}$ glauben. Davon sprich ausdrücklich der Text des 1 Joh 2, 4-7. Man soll dem Wort Gottes gehorchen um die Gebote auszuführen. Gehorsam sein, heisst glauben und glauben heisst lieben. Die Nächstenliebe ist also eine Frucht des Glaubens, sofern sie den Empfang des Lichts der Offenbarung Gottes bedeutet. Der durch die Offenbarung aufgeklärte Mensch kann und s oll lieben.

Schreiten wir noch weiter und stellen die Frage, wie das Verhältnis der Einhaltung der Gebote zu der Gottesgemeinschaft ist. Der Ausdruck „die Gebote einhalten" hat seine Entsprechung in „seine Gebote erfüllen”. 2 Joh 6 präzisiert: „wandeln nach seinen Geboten”. Ausserdem, wie wir im 1 Joh 2, 5 sahen, ,die Gebote einhalten” bedeutet ,sein Wort halten". So führt die Einhaltung der Gebote zum Leben nach Gottes Offenbarung und dies gibt die Sicherheit, in Gemeinschaft mit Gott zu bleiben. Das alles erlaubt uns folgende Folgerung $\mathrm{zu}$ formulieren: die Einhaltung der Gebote ist ein $\mathrm{Kr}$ iteri u m, das unsere Gemeinschaft mit Gott feststellen lässt. Weiter ist diese Gemeinschaft mit Vater und Sohn ein vahres Fundament in sich und ein Lebensprinzip der Einhaltung der Gebote.

Hinsichtlich der literarischen Struktur wurde dieses Kriterium in der charakteristischen Johannes Formel ausgedrückt: en toutō ginōskomen - „daran erkennen wir”. Die Einhaltung der Gebote ist nicht nur das Kriterium unserer Verbindung mit Gott, sondern auch die Wirklichkeit - ein Z e i chen, das sich vor allem in der brüderlichen Liebe ausdrückt: Agape der Kinder Gottes ist weiter ein Kriterium der Einhaltung der Gebote (vgl. 1 Joh 5, 2). Diese Wirklichkeit ist schon bekannt: bemerken wir den Gebrauch von Perfektum egnōkamen im $1 \mathrm{Joh}$ 3, 16 - die Folge dieser Erkenntnis dauert weiter. Diese Wirklichkeit weist darauf hin, dass Agape ein Ausgangspunkt für die Erreichung der wahren Gemeinschaft ist; es geht nämlich um diese Liebe, die vom Gott stammt und die uns zu Kindern Gottes macht (vgl. 1 Joh 3, 1).

Die Einhaltung der Gebote wird in der Auffassung des 1 Joh nicht in die juristische, sondern ausschliesslich in die moralische und in diesem Falle in die christliche Perspektive einbezogen. Es lohnt sich bei Gelegenheit die Worte vom 1 Joh 5, 3 zu vermerken: ,und seine Gebote sind nicht schwer", die zweifellos eine Anspielung an die Worte Jesu (Matth 23, 4 und 11, 30) sind und sich deutlich von den Rabinerdisputen zum Thema der schweren und leichten Gebote separieren. Es ist möglich, dass der Terminus nomos eben aus diesem Grunde im Johannesbrief nie gebraucht wurde. Agape ist also Wirklichkeit - Zeichen der 
Gemeinschaft mit dem Vater und Sohn. Und diese Gemeinschaft ist die Grundlage für die Einhaltung der Gebote, vor allem des Gebotes der Nächstenliebe. Diese gegenseitige Beziehung zwischen Agape und der Gemeinschaft mit Gott schliesst sich aber nich in sich: sie hat ihren Ursprung im Gott und ist für den Sieg durch die Erlösung Christi offen. Dieser Sieg ist der Glaube, durch den die Offenbarung Gottes und zwar durch Christi, der das Licht ist, aufgenommen wird und wodurch wir selbst Kinder des Lichtes werden (vgl. Joh 12, 36).

\section{DAS DOPPELGEBOT DER LIEBE - DAS MODELL DER KOMMUNIZIERENDEN GEFASSE}

Die Johannesbriefe aller Schriften des Neuen Testaments bedienen sich am häufigsten, proportionell zu ihrem Umfang - der Terminologie der Agape. Sie enthalten nämlich 31 auf 141 Erwähnungen des Verbs agapan (d. h. $22 \%$ aller Erwähnungen dieses Verbs im Neuen Testament), und 21 auf 116 Erwähnungen des Substantives agapē (d. h. 18\%).

Die Modelle des Doppelgebotes der Gottes- und Nächstenliebe, die in den biblischen Traditionen vorgeschlagen wurden, besitzen schon eine umfangreiche Literatur ${ }^{12}$. Wir werden es hier nicht besprechen und wollen uns auf die Feststellung beschränken, dass es in der synoptischen Tradition verschiedene Versuche gibt, die zwei Gebote der Liebe miteinander in Einklang zu bringen. Der Versuch von Markus (12, 28-34), der älteste, muss im Kontext der Missionsverhältnisse der werdenden Kirche betrachtet werden. Diese Perikope von Markus wurde schon von Spicq als Muster der biblischen Theologie charakterisiert ${ }^{13}$. Auf dieselbe Weise die Perikope von Matthäus (22, 34-40). Es scheint auch, dass Lukas Perikope (10,25-28) im katechetischen Kontext der allmählichen Belehrung der Christus Schüller betrachtet werden kann.

Wollen wir kurz die Johannes Tradition erforschen, die sich auf das Verhältnis zwischen der Gottes- und der Nächstenliebe bezieht, eine Tradition, die sich wohl am besten in seinem ersten Brief wiederspiegelt.

Die formale Zusammensetzung zweier Lieben erfolgt im 1 Joh 2, 29 - 4,6. Den Ausgangspunkt bildet hier die Liebe, die uns von dem Vater erwiesen wird, dank der wir mit Ihm in Verbindung stehen $(3,1)$.

12 Vgl. aus der reichen Literatur: A. Niss e n, Gott und der Nächste im antiken Judentum. Untersuchungen zum Doppelgebot der Liebe (Wiss. Untersuch. zum NT 15), Tübingen 1974; R. H. Fulle r, Das Doppelgebot der Liebe, in: Jesus Christus und Theologie. Neutestamentliche Festschrift für $\mathrm{H}$. Conzelmann zum 60 . Geburstag. Hrsgbn. G. Stre $\mathrm{cker}$, Tübingen 1975; J. Chmiel, Le rapport entre l'amour de Dieu et l'amour du prochain dans la tradition biblique. (franz. Zusammenfass.), in: „Analecta Cracoviensia” 1 (1969) 106-119.

13 Agapè dans le NT, I, 89. 
Zwecks formalen Verschmelzung beider Gebote in eins werden von dem Autor des Briefes einige verwandten Themen angeführt:

- Kain und sein Bruder $(3,12)$,

- das Herz und die Liebe Gottes $(3,17)$,

- lieben in Tat und Wahrheit $(3,18)$.

Kain wird zum Archetyp des Hasses, so wie sein Bruder, Abel, zum Archetyp der Liebe wird. An Abels Stelle erscheint in V. $16 \mathrm{Er}$ - ekeinos - Jesus Christus, der zum neuen Archetyp der brüderlichen Liebe wird und den wir nachahmen sollen. Wie es schon sehr treffend bemerkt wurde, wurzelt im Alten Testament die brüderliche Liebe durch die Idee der Nachahmung in der Gottesliebe. Dies erinnert uns an die Genesisgeschichte von der Schöpfung. Andererseits wird im Johannes Text unterstrichen, dass wir nur durch das Kreuzopfer zum Verständnis kommen, was die Liebe ist $(3,16)$. So werden von dem Autor des Briefes die brüderliche Liebe und die Liebe Gottes in der Perspektive der Schöpfungs- und Erlösungstheologie nahegebracht.

Indem Johannes ta splagchna „Herz” $(3,17)$ sagt, denkt er an Liebes- und Mitleidsgefühl: es geht hier um ein, den bedürftigen Brüdern gegenüber, barmherziges Herz. Mit dieser Barmherzigkeit verbindet sich die Liebe Gottes, der in uns wohnt. Das Themenensemble: Liebe Gottes - barmherziges Herz, das in den Schriften das Neuen Testaments erwähnt wird, bekommt im Johannesbrief eine theologische Perspektive. Zuerst wird die Liebe Gottes dem menschlichen Gefühl begegnet, was aber nichts von der Laune oder Mode in sich haben darf, sonderm eine tiefe Liebe darstellen, die in dieser Liebe wurzelt, die Christus erwiesen hat als er am Kreuz starb.

Die Formel im 1 Joh 3, 18: „lieben in Tat und Wahrheit” verbindet in sich das vorhererscheinende Thema der Barmherzigkeit mit dem Thema der Wahrheit. Das Thema der Wahrheit (alētheia) bei hl. Johannes gehört zu den Offenbarungsthemen. Im 3, 19 präzisiert Johannes unser existentielle Verhältnis zur Offenbarung: „wir sind aus der Wahrheit”. Die Nächstenliebe soll aktiv sein, sich in Tat ausdrücken, obwohl sie sich andererseits nicht nur mit ihrem menschlichen Aspekt, auch wenn der sehr intesiv wäre, begnügen darf. Die Nächstenliebe muss aus der lebendigen Quelle der göttlichen Offenbarung schöpfen, aus dieser Wahrheit, die das offenbarte Wort darstellt (theologischer Aspekt). Wenn unsere Liebe wahr ist, soll sie eine Hingabe des Sich selbst sein: „und auch wir sind verpflichtet, für die Brüder das Leben hinzugeben" $(3,16 \mathrm{~b})$. Hier gibt es wieder einen Punkt, in dem sich die Liebe Gottes mit der Nächstenliebe kreuzt. Das Leben zu opfern bedeutet nicht immer eines Märtyrertodes zu sterben, sondern in dem Geiste zu leben, der den Herren belebte, als er für uns Sein Leben gab. Lieben heisst sein Leben zu 
geben, das heisst in dem Nächsten Christus zu sehen und Ihm zu dienen, indem man Ihm das Beste aus dem Leben gibt (der christozentrische Aspekt).

Im 1 Joh 3, 23-24 kommt noch der Begriff des Gebotes hinzu, der stilistisch wie eine Wortklammer die zwei Begriffe der Liebe miteinander verbindet. Und so haben wir in einem Gebot zwei Pflichten:

- im Namen Jesus Christi zu glauben,

- einander lieben.

Hier lässt sich der Einfluss der Weisheitstradition erkennen: die Einhaltung der Gebote ist Ausdruck der Liebe Gottes. Die Liebe Gottes, die durch die Einhaltung der Gebote bewiesen wird, verbindet sich mit der Menschenliebe - der christologische Glaube verleiht dieser Verbindung einen neuen Charakter.

Im 1 Joh 4, 7-21 wird von dem Autor die Relation der brüderlichen Liebe zu dem Liebesfundament, dem Gott, dargestellt — „Gott ist die Liebe" $(4,8.16)$ : die brüderliche Liebe verbindet sich mit der Anwesenheit Gottes in uns $(4,12.16)$. Der Ausgangspunkt wird immer die erste und uneigennützige Liebe Gottes zu uns sein $(4,19)$. Im 5,2 ist die Gottesliebe das Kriterium der Nächstenliebe: „Daran erkennen wir, dass wir die Kinder Gottes lieben, wenn wir Gott lieben und seine Gebote ausführen".

Um es zusammenzufassen: der hl. Johannes identifiziert die Liebe zum Gott nicht restlos mit der Nächstenliebe. Er stellt nur die Relation zwischen ihnen fest und dies auf eine Weise, die wir ohne Überfreibung als die radikalste und glücklichste nennen könnten. Das Wesen der Vereinigung der Liebe in Johannes Konzeption beruht auf:

1) der Unterstreichung der Offenbarung der Gottesliebe an den Menschen: in diesem Sinne hat die Gottesliebe den Vorrang vor der Nächstenliebe $(4,7-12.19)$;

2) die Nächstenliebe ist organisch mit der Gottesliebe verbunden, so dass die Liebe Gottes und der Menschen derselben Natur ist: es ist eine offenbarte Liebe $(4,21)$;

3) wir können Gott lieben nur indem wir die Menschen lieben. In diesem Sinn kann man von dem Primat der Nächstenliebe über die Gottesliebe sprechen.

In der theologischen Konzeption vom hl. Johannes stellen die Gottesliebe und die Nächstenliebe ein System der kommunizierenden Gefässe dar: die Liebe verursacht die Nächstenliebe und umgekehrt. 
IV. EPIPHANIE DER AGAPE - DIE VERTIKALE DIMENSION DER LIEBE

Das Einhalten der Gebote in Johannes Konzeption - wie wir das verfolgen konnten - wurde in die Perspective Gottes, der Licht und Liebe ist, gestellt ${ }^{14}$. Das Gebot der Liebe entspringt der schöpferischen Kraft der Agape Gottes. Die Einhaltung der Gebote erlaubt uns im Lichte zu sein und hütet uns vor dem Verfall in die Dunkelheit. Die Nächstenliebe, wie alles, was geschaffen wurde, verdankt ihr Dasein der Offenbarung der Liebe Gottes.

Der göttliche Ursprung der Liebe wird im 1 Joh mit der Formel ek tou Theou estin - „die Liebe ist aus Gott” $(4,7 \mathrm{a})$ angedeutet. Diese Feststellung wird durch eine andere verstärkt, indem man sagt, dass die Gottesgabe der Agape zu unserem an Sohnesstalt Annehmen durch Gott und zu unserer Erkenntnis des Gottes gehört: „und jeder, der liebt, ist aus Gott gezeugt und kennt Gott" (4, 7b). Das Prinzip der Nächste̊nliebe ist also, vom Gott gezeugt zu werden und Gott zu erkennen. Die Formel „,aus Gott gezeugt” ist mit der Formel „,aus Gott sein" parallel und drückt ein Ursprungsfaktum in der Offenbarung des neuen Lebens aus. Gott zeugte die Christen indem er ehnen seine Natur und sein Leben kommunizierte. Verfolgen wir die Zusammensetzung: gezeugt - kennt. Das Perfektum Passivi gegennètai bedeutet, dass die Geburt aus Gott ein schon vorhandener Zustand sei; das Präsens ginōskei, das dem agapōm entspricht, betont, dass der, der liebt, die Erkenntnis auf eine kontinuierliche Weise besitzt; der Aorist egnō drückt aus, dass der, der nicht liebt, zu der Erkenntnis noch nicht gekommen ist.

Hl. Johannes betont: „daran haben wir die Liebe erkannt” $(3,16)$ und will damit sagen, dass es hier um die Erkenntnis der Offenbarung der Liebe durch den Glauben geht. Die Nächstenliebe verursacht das Steigen der Erkenntnis Gottes, um zu lieben jedoch, muss man aus Gott sein und beginnen Gott zu erkennen. Wenn uns die Gotteskindheit auf diese Weise vom Gott vermittelt wurde, dass wir sie erkennen können, so gibt sie uns auch die Fähigkeit der Liebe.

Agape ist ein Objekt der Botschaft (1 Joh 3,11). Diese Liebe hat sich dem Menschen offenbart; dies wurde mit Hilfe des Wortes phaneroun ausgedrückt: „darin ist die Liebe Gottes unter uns zu erschienen” (4, 9). Das Wort phaneroun wird im 1 Joh neun Mal gebraucht. Es geht hier um die Erscheinung Christus - Leben - Liebe, und es wurde durch den Aorist Passivi ephanerōthē ausgedrückt, weil Gott ständig seine Liebe erwiesen hatte. Der 1 Joh 4, 10 präzisiert weiter das Wesen der Erscheinung der Liebe: „darin besteht die Liebe, nicht dass wir Gott

${ }_{14}$ Vgl. J. C hmi e l, Lumière et charité d'après la première épitre de saint Jean, Rome 1971. 
geliebt haben, sondern dass er uns geliebt und seinen Sohn als Sühne für unsere Sünden gesandt hat". Die Aussage des 9. und 10. Verses ist folgend: die Liebe Gottes zu zeigen ist die Sendung des einziggezeugten Sohnes in die Welt. Eben in der Kenosis Christi wird das Geheimnis der Liebe offenbart.

Das alles wird im historischen und theologischen Aspekt der Offenbarung der Gottesliebe unterstrichen. Die historische Tatsache ist die erlösende Menschwerdung, die aus dem höchsten Punkt die Grösse der Gottesliebe in ihrer endgültigen theologischen Vollendung zeigt. Diese theologische Vollendung ist die Vollkommenheit der Liebe:

„seine Liebe ist in uns vollendet" $(4,12)$. Daran erinnert uns Joh 13,1:

"(Jesus) liebte sie bis zum Ende (eis telos)".

Die Vollkommenheit der Liebe hat ihren gemeinschaftlichen "Aspekt: die Nächstenliebe, in der sich die Gemeinschaft mit Gott realisiert. Dieser Aspekt erscheint auch auf der theologischen Ebene: als Besitz des Lebens Gottes, und auf der ekklesialen Ebene: als Apostelgemeinschaft. In diesem Sinne ist die Vollendung der Liebe in Apostelvoraussage vorhanden: so ist die Bedéutung der griech. aggelia: „die Botschaft”.

Die Vollkommenheit der Liebe ist eine dynamische Bewegung. Sehr treffend drück es Joh 17, 23 aus, indem er drei Elemente zusammenstellt: lieben - senden - vollenden: „Ich in ihnen und du in mir, so mögen sie zur vollendeten Einheit gelanden, damit die Welt erkenne, dass du mich gesandt und sie geliebt hast, wie du mich geliebt hast."

Johannes Konzeption der Vollendung der Liebe ist einzig und einmalig, vielleicht inspirierte sie das alte eucharistische Didache Gebet: „Recordare, Domine, ecclesiae tuae, ut eam liberes ab omni malo eamque perficias in caritate tua (teleiosai en tè agapé - 10,5)". Man nennt mit Recht die Konzeption der Agape - Christologie der Liebe.

Als Schlussfolgerung der obigen Erwägungen möchte ich folgende Bemerkung formulieren.

Wenn wir die christliche Ethik auf dem Agape-Begriff stützen wollen, soll das offenbarte Modell gefunden werden. So ein offenbartes Modell der Liebe Gottes und des Nächsten scheint der Erste Johannesbrief zu sein, nicht durch die Angabe der Erscheinung des Begriffes der Agape, sondern durch die Hineinsetzung dessen in den Kontext des Lebens der Urgemeinschaft der Gläubigen, die die Gottes- und Nächstenliebe erfuhren. Es ist nicht nur eine Beschreibung der erlebten Erfahrung, sondern vor allem eine Botschaft für uns: „was wir gesehen und gehört haben, das verkündigen wir auch euch, damit auch ihr Gemeinschaft habet mit uns" (1 Joh 1, 3). 